\title{
Search for SUSY at ATLAS
}

\author{
Iris Borjanović (for the ATLAS collaboration)* ${ }^{* \dagger}$ \\ Institute of Physics Belgrade \\ E-mail: iris@ipb.ac.rs
}

The search for physics beyond the Standard Model is one of the main goals of the general purpose ATLAS detector at Large Hadron Collider at CERN. This report summarizes the prospects of the ATLAS detector to find experimental evidence for R-parity conserving Supersymmetry in channels with large missing transverse energy, multiple high energy jets and eventually leptons for both $14 \mathrm{TeV}$ and $10 \mathrm{TeV}$ centre-of-mass energies. The $5 \sigma$ discovery reach was addressed with systematic uncertainties included. Supersymmetric particles mass measurements and determination of minimal Supergravity model parameters are also discussed.

XVIII International Workshop on Deep-Inelastic Scattering and Related Subjects

April 19 -23, 2010

Convitto della Calza, Firenze, Italy

\footnotetext{
* Speaker.

${ }^{\dagger}$ Many thanks to the organizing committee of the DIS2010 workshop and to everyone in the ATLAS collaboration whose work contributed to this paper. The work of this author is supported by Serbian MNTR (project 141037).
} 


\section{Introduction}

Supersymmetry (SUSY) [1] is one of the most attractive extensions of the Standard Model. Supersymmetry predicts that every Standard Model particle has its supersymmetric partner whose spin differs by $1 / 2$. Since supersymmetric particles have not been discovered yet, it is supposed that they are heavy and that supersymmetry is broken. One mechanism of supersymmetry breaking is the minimal Supergravity model (mSUGRA). Within mSUGRA model all masses, mixings and decays of SUSY and Higgs particles are determined in terms of four characteristic parameters and a sign: the scalar mass $m_{0}$, the gaugino mass $m_{1 / 2}$, the trilinear coupling $A_{0}$, the ratio of the Higgs vacuum expectation values $\tan \beta$ and the sign of the supersymmteric Higgs mass parameter $\mu$. In mSUGRA model R-parity is conserved. As a consequence SUSY particles are always produced in pairs and at the end of the decay chain of every SUSY particle is the lightest supersymmetric particle (LSP), which is stable, massive, neutral, weakly interacting and thus undetectable.

Search for the Supersymmetric particles is one of the most important aims of the ATLAS experiment at Large Hadron Collider (LHC) at CERN, which started data taking at the end of 2009. ATLAS made a lot of effort in the previous few years in order to prepare for the analysis of the first LHC data. This report presents the results from the Computing System Commissioning (CSC) exercise [2], normalised to the LHC integrated luminosity of $1 \mathrm{fb}^{-1}$ at the center of mass energy

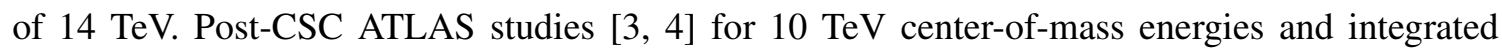
luminosity of $200 \mathrm{pb}^{-1}$ are also discussed. We focus on the mSUGRA model here.

\section{Inclusive searches}

It is expected that squarks and gluinos will be dominantly produced in the initial protonproton interactions at LHC and that their cascade decays will give high transverse momenta jets, leptons and LSP particles in the final states. Due to the presence of the undetectable LSP particle, the lightest neutralino $\tilde{\chi}_{1}^{0}$, there will be a large missing transverse energy $E_{T}^{\text {miss }}$ in the event. ATLAS have analyzed [2, 3] various channels with 2,3 or 4 jets and 0,1 or 2 leptons. The study presented here was performed on the selected set of benchmark points in the mSUGRA five dimensional parameter space. Event selection was performed by applying cuts on the number and transverse momenta of hadronic jets, missing transverse energy, lepton transverse momenta and sphericity of event. For the one-lepton channel additional cut is applied on transverse mass $M_{T}=\sqrt{2\left(p_{T}^{l e p} E_{T}^{\text {miss }}\left(1-\cos \left(\Delta \Phi\left(l e p, E_{T}^{\text {miss }}\right)\right)\right)\right.}$ formed from the missing transverse energy and the identified lepton. The effective mass $M_{\text {eff }}$ is used to check whether there is an excess of SUSY over Standard Model events. The effective mass is defined as the sum of the missing transverse energy, transverse momenta of the most energetic jets and transverse momenta of all leptons in the event, $M_{\text {eff }}=E_{T}^{\text {miss }}+\sum_{i=1}^{N_{\text {jets }}} p_{T}^{\text {jet }, i}+\sum_{i=1}^{N_{\text {lep }}} p_{T}^{\text {lep }, i}\left(N_{\text {jets }}\right.$ is the number of jets (2-4) and $N_{\text {lep }}$ is the number of leptons (0-2)). The effective mass also gives an indication of the SUSY mass scale. The 0-lepton mode is the most generic one, but it is sensitive to the background from mismeasured QCD multijet events. Asking one lepton in addition reduces QCD background and the 1-lepton mode is often considered to be the golden mode for early SUSY searches. Figure 1 1 shows the effective mass $M_{\text {eff }}$ distributions for the mSUGRA benchmark point SU4 $\left(m_{0}=200 \mathrm{GeV}, m_{1 / 2}=160\right.$ $\mathrm{GeV}, A_{0}=-400, \tan \beta=10, \operatorname{sign}(\mu)=+$ ) and the Standard Model background for an integrated 

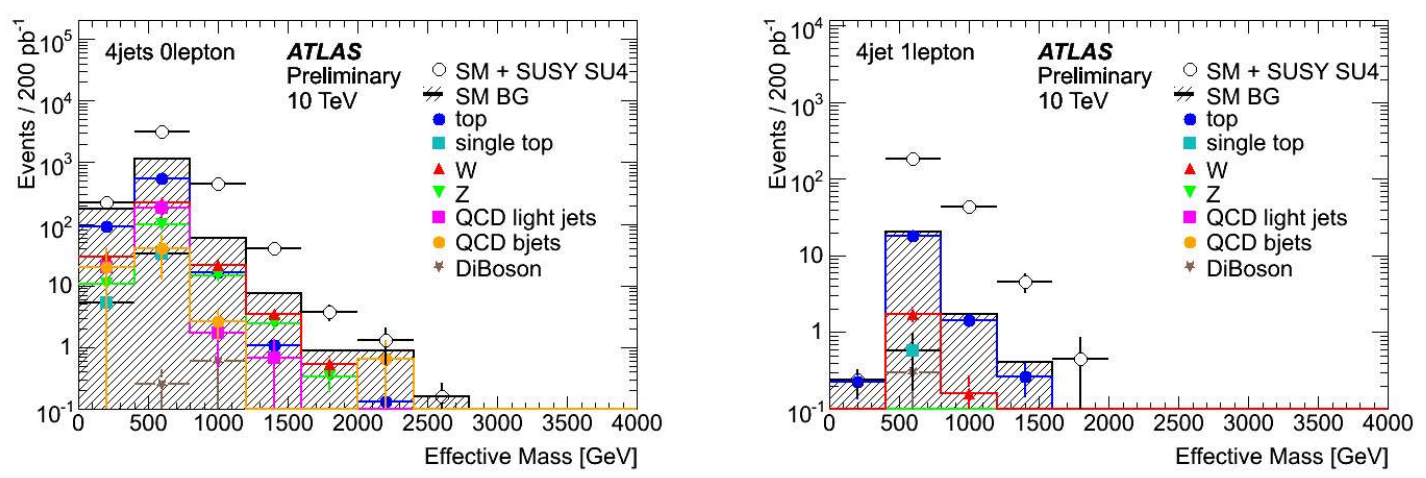

Figure 1: $M_{\text {eff }}$ distribution in the 0-lepton channel (left) and 1-lepton channel (right) of the SUSY inclusive searches for an integrated luminosity of $200 \mathrm{pb}^{-1}$ at $10 \mathrm{TeV}$. The open circles shows the signal (ATLAS mSUGRA benchmark point SU4) and Standard Model background summed together, while the shaded area shows the total Standard Model background. The legend gives the components of the Standard Model background.

luminosity of $200 \mathrm{pb}^{-1}$ at $10 \mathrm{TeV}$ in the channel with 0-leptons and 4-jets and in the channel with 1-lepton and 4-jets (ATLAS study [3]). Clear excess of SUSY over Standard Model background events is visible in both distributions. The squark and gluino masses for the SU4 point are the following: $m(\tilde{g})=413.37 \mathrm{GeV}, m\left(\tilde{d}_{L}\right)=419.84 \mathrm{GeV}, m\left(\tilde{u}_{L}\right)=412.25 \mathrm{GeV}, m\left(\tilde{b}_{1}\right)=358.49$ $\mathrm{GeV}, m\left(\tilde{t}_{1}\right)=206.04 \mathrm{GeV}, m\left(\tilde{d}_{R}\right)=406.22 \mathrm{GeV}, m\left(\tilde{d}_{R}\right)=404.92 \mathrm{GeV}, m\left(\tilde{b}_{2}\right)=399.18 \mathrm{GeV}$, $m\left(\tilde{t}_{2}\right)=445.00 \mathrm{GeV}$.

The same-charge dilepton channel with jets and missing transverse energy, described in details in ATLAS study [ $₫$ ], is also a powerful channel for early SUSY discovery and is almost background free. Data-driven method was used in order to estimate Standard Model background in this channel. After event preselection cuts (two leptons with $p_{T}^{l 1}>20 \mathrm{GeV}, p_{T}^{l 2}>10 \mathrm{GeV}$ and invariant mass $m_{l l}>5 \mathrm{GeV}$, at least two jets with $p_{T}>40 \mathrm{GeV}$ and $E_{T}^{\text {miss }}>50 \mathrm{GeV}$ ) remaining signal and background processes are classified into four different categories by using four variables: $E_{T}^{\text {miss }}$, transverse momentum of the second leading lepton $p_{T}^{l 2}$, transverse momentum of the second leading jet $p_{T}^{j 2}$ and transverse mass $M_{T}$. Regions are selected as follows: signal region SR $\left(E_{T}^{\text {miss }}>80 \mathrm{GeV}\right.$, $\left.p_{T}^{j 2}>80 \mathrm{GeV}\right)$, sideband region $\mathrm{SB}\left(50<E_{T}^{\text {miss }}<80 \mathrm{GeV}, 40<p_{T}^{j 2}<80 \mathrm{GeV}\right)$, A region $\left(p_{T}^{l 2}>\right.$ $\left.20 \mathrm{GeV}, M_{T}>80 \mathrm{GeV}\right)$ and $\mathrm{B}$ region $\left(10<p_{T}^{l 2}<20 \mathrm{GeV}, 50<M_{T}<80 \mathrm{GeV}\right)$. The purpose of this selection was to estimate the number of background events in the region dominated by the signal events $A_{S R}$, by using extrapolation method: $\frac{A_{S B}}{B_{S B}} \simeq \frac{A_{S R}}{B_{S R}}$. It was concluded that for the benchmark point SU4 clear signature can be observed with an integrated luminosity of $200 \mathrm{pb}^{-1}$ at $10 \mathrm{TeV}$.

A scan of parameter space was performed [2 -4 ] in order to explore a $5 \sigma$ discovery reach of different SUSY models. Figure 2 shows the $5 \sigma$ discovery reach as a function of $m_{0}$ and $m_{1 / 2}$ for $\tan \beta=10$ mSUGRA scan for the channels with 0,1 and 2 leptons. Results obtained for the integrated luminosity $\mathrm{L}=1 \mathrm{fb}^{-1}$ and $14 \mathrm{TeV}$ are displayed on the left, while the results for the integrated luminosity $\mathrm{L}=200 \mathrm{pb}^{-1}$ at $10 \mathrm{TeV}$ are on the right. For the $14 \mathrm{TeV}$ analysis [D] with 1 $\mathrm{fb}^{-1}$ of data a systematic error of $50 \%$ for QCD background and $20 \%$ for $W, Z$ and $t$ background is estimated by using data-driven method. The best reach with squark and gluino masses up to 1.5 $\mathrm{TeV}$ is obtained in 0-lepton channel. In the 1-lepton channel, which is more robust against QCD 

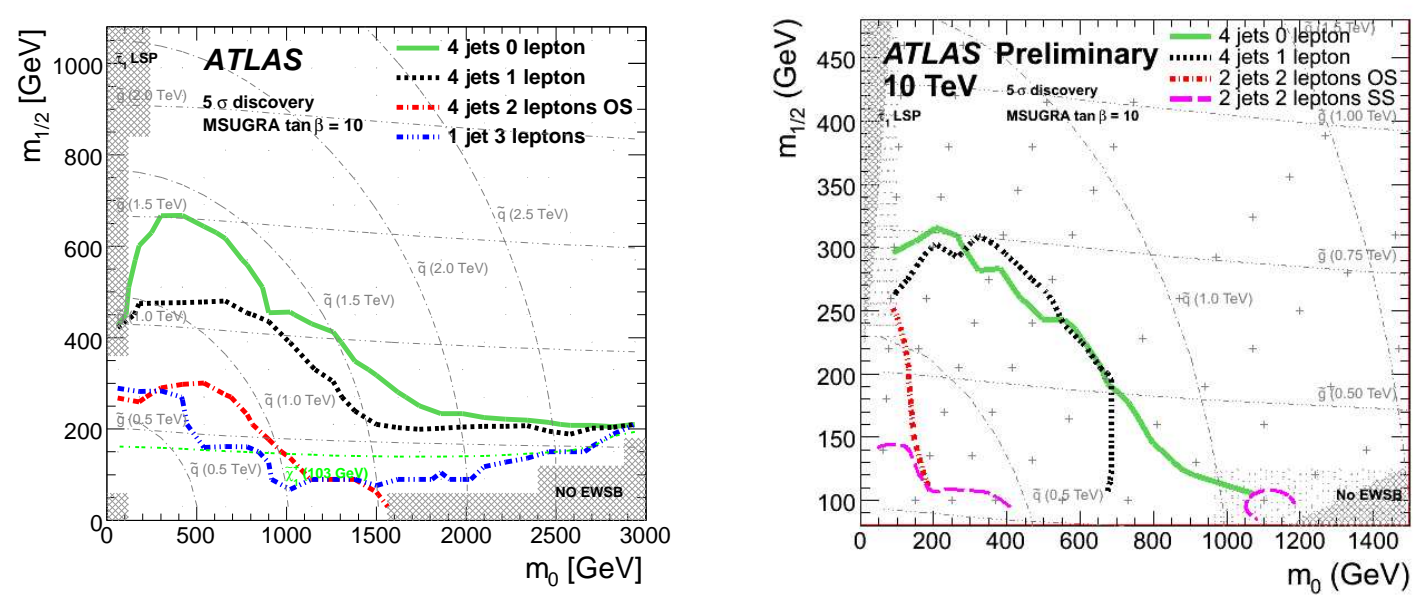

Figure 2: $5 \sigma$ discovery reach as a function of $m_{0}$ and $m_{1 / 2}$ for $\tan \beta=10$ mSUGRA scan for the channels with 0,1 and 2 leptons. Left: integrated luminosity $\mathrm{L}=1 \mathrm{fb}^{-1}$ and $14 \mathrm{TeV}$ center-of-mass energy. Right: integrated luminosity $\mathrm{L}=200 \mathrm{pb}^{-1}$ at $10 \mathrm{TeV}$.

background, masses close to $1 \mathrm{TeV}$ are attainable. For the $10 \mathrm{TeV}$ studies [3, 4 and integrated luminosity of $\mathrm{L}=200 \mathrm{pb}^{-1}$, a systematic uncertainty of $50 \%$ is assumed. In this case the 0 -lepton and 1-lepton channels have similar discovery reach with SUSY masses up to 600-700 GeV.

\section{Mass and parameter measurements}

After SUSY signatures have been established, it is crucial to measure masses and spins of the new particles and to obtain underlying model parameters. Due to the presence of the undetectable lightest supersymmetric particles $\tilde{\chi}_{1}^{0}$ in the final state of every SUSY decay, direct mass reconstruction is not possible. The distributions of the invariant masses formed from the detectable final state particles have kinematic endpoints that can be fitted and used for extraction of SUSY masses. The theoretical positions of the endpoints can be calculated from the conservations of energy and momentum. From the left squark cascade decay [2] $\tilde{q}_{L} \rightarrow \tilde{\chi}_{2}^{0} q \rightarrow \tilde{l}_{R}{ }^{\mp} l^{\mp} q \rightarrow l^{+} l^{-} q \tilde{\chi}_{1}^{0}$ (where $\left.\tilde{q}_{L}=\tilde{u}_{L}, \tilde{d}_{L}, \tilde{s}_{L}, \tilde{c}_{L}, \tilde{l}=\tilde{e}, \tilde{\mu}, \tilde{\tau}\right)$ a few invariant masses can be reconstructed: dilepton invariant mass $m_{l l}$, lepton-lepton-jet invariant mass $m_{l l j}$ and two lepton-jet invariant masses $m_{l j(h i g h)}$ and $m_{l j(l o w)}$. The label low/high indicates minimum/maximum of the two masses $m_{l^{-} j}$ and $m_{l^{+} j}$. Combinatorial SUSY and Standard Model background can be suppressed effectively by using flavour subtraction. For the SU3 mSUGRA benchmark point $\left(m_{0}=100 \mathrm{GeV}, m_{1 / 2}=300 \mathrm{GeV}, A_{0}=-300, \tan \beta=6\right.$, $\operatorname{sign} \mu=+$ ) the fitted endpoints of the reconstructed invariant mass distributions together with their theoretical values are shown in Table 1 , for the integrated luminosity $\mathrm{L}=1 \mathrm{fb}^{-1}$ at $14 \mathrm{TeV}$. The first error is statistical, the second and third are the lepton energy scale uncertainty and the jet energy scale uncertainty, respectively. Labels $\min$ and $\max$ of the endpoints denote kinematic minimum and maximum of the mass distributions. All measured endpoints are compatible with their true values. Kinematic endpoints of mass distributions formed with jets have larger uncertainties due to the fact that among all jet permutations, the wrong jet might be selected. e The mSUGRA model parameters can be obtained from the set of measured endpoints [2]. For the SU3 point $m_{0}$ and 


\begin{tabular}{c|c|c}
\hline Endpoints & SU3 Measured $(\mathrm{GeV})$ & SU3 Truth $(\mathrm{GeV})$ \\
\hline$m_{l l}^{\max }$ & $99.7 \pm 1.4 \pm 0.3$ & 100.2 \\
$m_{l l j}^{\max }$ & $517 \pm 30 \pm 10 \pm 13$ & 501 \\
$m_{l l j}^{\min }$ & $265 \pm 17 \pm 15 \pm 7$ & 249 \\
$m_{l j(\text { mow })}^{\max }$ & $333 \pm 6 \pm 6 \pm 8$ & 325 \\
$m_{l j(\text { high })}^{\max }$ & $445 \pm 11 \pm 11 \pm 11$ & 418 \\
\hline
\end{tabular}

Table 1: Measured endpoint values for the mSUGRA benchmark point SU3 with an integrated luminosity $\mathrm{L}=1 \mathrm{fb}^{-1}$ at $14 \mathrm{TeV}$. The truth values are also listed.

$m_{1 / 2}$ parameters can be derived with a precision better then $10 \%$ already with $1 \mathrm{fb}^{-1}$ of data. The determination of the parameters $\tan \beta$ and $A_{0}$ is more complicated since no information from the Higgs sector is available at low luminosity.

\section{Conclusions}

If SUSY masses are within sub-TeV range the ATLAS experiment will be sensitive to early SUSY searches described by R-parity conserving models at $10 \mathrm{TeV}$ center-of-mass energy already with integrated luminosity of $200 \mathrm{pb}^{-1}$. Understanding the detector performance and controlling the Standard Model background are important goals of early SUSY analysis. Once a signal is established, the kinematic endpoints reconstructed in the specific SUSY decay chains will be used to extract corresponding SUSY masses.

\section{References}

[1] S.P. Martin, A Supersymmetry Primer, 1997 [hep-ph/9709356].

[2] ATLAS Collaboration, Expected Performance of the ATLAS Experiment, Detector, Trigger and Physics, Report CERN-OPEN-2008-020, Geneva, 2008 [arxiv: 0901.0512$].$

[3] ATLAS Collaboration, Prospects for Supersymmetry and Universal Extra Dimensions discovery based on inclusive searches at a $10 \mathrm{TeV}$ center-of-mass energy with the ATLAS detector, Atlas Note ATL-PHYS-PUB-2009-084, Geneva, 2009.

[4] ATLAS collaboration, Searching for Supersymmetry with two same-sign leptons, multi-jets plus missing transverse energy in ATLAS at $\sqrt{s}=10 \mathrm{TeV}$, ATLAS Note ATL-PHYS-PUB-2009-085, Geneva, 2009. 